\title{
REFLEXIONES ACERCA DE LA DEFINICIÓN DE RADICACIÓN Y SU RELACIÓN CON LA CONSTRUCCIÓN DE NUEVOS CONCEPTOS
}

\author{
Fernando Benítez y Marilina Carena
}

\begin{abstract}
RESUMEN. En este trabajo presentamos experiencias transitadas por cada uno de los autores en distintos niveles de educación, referidas a un mismo concepto: la radicación. Tanto en diferentes grupos de la escuela secundaria como en ingresantes a la universidad hemos observado ciertos errores recurrentes de conceptos que luego redundan en dificultades o contradicciones en la construcción de nuevos objetos matemáticos. La repetición de dichos errores nos condujo a revisar algunos libros escolares, evidenciando la importancia de la labor del docente para lograr erradicarlos, desde el cuestionamiento y debate continuo del material bibliográfico, y desde la precisión y consistencia en la presentación de los conceptos.
\end{abstract}

\begin{abstract}
AвSTRACт. In this work we present some experiences that both authors have witnessed in different educational levels, regarding the same concept: the nth root. We have observed certain recurrent errors of concepts, not only in high school students but also in college students, that later conduct to difficulties or contradictions in the construction of new mathematical objects. The recurrence of these errors led us to review some school books, where the importance of the teacher's work becomes essential to eradicate possible errors, from the continuous questioning and debate of the bibliographic material, and from the precision and consistency in the presentation of the concepts.
\end{abstract}

\section{Introducción}

Quienes estamos en contacto con la Matemática, ya sea estudiándola o transmitiéndola, no tenemos dudas acerca de su consistencia, coherencia y su rigurosidad. La entendemos como una construcción constante de conocimientos mediante razonamientos lógicos a partir de ciertos axiomas. En esta construcción, una condición necesaria para la validación y aceptación de nuevos conceptos y resultados es que no contradigan a otros presentados y probados previamente.

Palabras clave: Educación Matemática, Escuela Secundaria, Radicación.

Keywords: Mathematics Education, Secondary School, $n$th Root. 
Sin embargo, en el proceso de transmisión de los objetos culturales de la Matemática, encontramos que ciertos conceptos son presentados en algunos textos escolares de manera que atenta con esa consistencia, coherencia y rigurosidad esperadas, generando luego procedimientos algebraicos sin sentido y una inconsistencia entre los mismos objetos matemáticos, como quedará expuesto a lo largo del trabajo.

En particular, en este artículo centramos nuestra atención en contenidos relacionados a la radicación. Mostraremos, mediante ejemplos, algunas ambigüedades y errores provenientes de ciertos textos escolares, y cómo estos pueden transformarse en obstáculos que generan contradicciones en el aprendizaje de nuevos conceptos bien definidos. Así, nos hacemos eco de las palabras pronunciadas en (Bidart Gauna, Cabral, Cafure, Cambriglia, y Fuentes, 2017):

Entendemos que los libros de textos utilizados en la escuela media son, en cierto modo, los responsables de difundir estas malas interpretaciones. Esto nos lleva también a discutir sobre la formación de profesores: en qué medida los ámbitos de formación contribuyen a formar ideas erróneas en este contexto. $(p .9)$

Si bien los errores o ambigüedades presentadas en este trabajo no aparecen cuando se trabaja la radicación en el campo de los números naturales, los mismos aparecen inmediatamente cuando se extiende a los números enteros $\mathrm{y}$, en consecuencia, cuando se trabaja con números reales. Los textos escolares aquí mencionados corresponden a la operación en estos dos últimos conjuntos numéricos. No consideramos la radicación en los números complejos.

El objetivo de este trabajo es aportar observaciones que puedan colaborar en la labor del docente de detectar dichos errores, debatirlos, corregirlos y, así, erradicarlos. A su vez, ofrecemos un camino que permita abordar ciertos conceptos de manera coherente y consistente con eventuales aprendizajes posteriores.

\section{§1. La radicación}

En los primeros años de la escolaridad secundaria aprendimos que la radicación es una operación en la que intervienen dos números, $x$ y $n$, llamados radicando e índice, respectivamente, y que se escribe como $\sqrt[n]{x}$. El símbolo $\sqrt{ }$ se denomina radical.

En ese contexto, aprendimos también que si $n=2$ se lee "raíz cuadrada de" y que, por convención, no se escribe como índice del radical. Por ejemplo, $\sqrt{4}$ se lee "raíz cuadrada de 4". Resulta muy probable que muchas personas no vean nada erróneo en la siguiente afirmación:

$$
\sqrt{4}= \pm 2
$$


De hecho, en (Sobel y Lerner, 1996, p.38) se enuncia: “ ¿Dijo usted que $\sqrt{25}= \pm 5$ ? Si lo hizo, ¡cometió un error muy común!"

Aunque este error aparece en libros antiguos como el célebre "Tapia”, aún persiste en algunos actuales (ver, por ejemplo, (Covelo y Covelo, 2019, p.14) donde el concepto se aborda en el campo de los números reales; (Pacetti y Bonardi, 2016, p.19) o (Ferraris y Tasso, 2008, p.35)). En particular, en (Covelo y Covelo, 2019) se enuncia que $\sqrt{9}= \pm 3$, y se lo reescribe como

$$
\sqrt{9}=x, \quad \text { con }|x|=3 .
$$

Durante un taller para 25 docentes de escuelas primarias y secundarias, la segunda autora de este trabajo propuso una votación anónima, en la que debían colocar un papel en una urna marcando la opción que consideraban correcta para el valor de $\sqrt{4}$. Las dos opciones posibles eran $\sqrt{4}=2$ o $\sqrt{4}= \pm 2$. Por otro lado, propuso una encuesta a través de la cuenta de Instagram de su cátedra, de la que participaron voluntariamente 74 estudiantes universitarios de carreras denominadas "exactas", provenientes de distintas escuelas y ciudades. En ambos casos, el resultado llamó la atención: aproximadamente el 60 \% votó por la opción incorrecta $\sqrt{4}= \pm 2$.

Si bien la cantidad de personas encuestadas es baja como para establecer una conclusión al respecto o considerar la experiencia como un trabajo estadístico, nos motivó a abordar el origen de esta concepción equivocada y de qué manera puede afectar a la construcción del nuevo conocimiento.

\subsection{La definición}

Comencemos por mostrar algunos de los obstáculos que pueden surgir como consecuencia de la aceptación del error conceptual presentado al comienzo.

Con el objetivo de estudiar la jerarquía de las operaciones, es común que se propongan ejercicios combinados en los cuales se presente la radicación. Supongamos que se llega a esto con la idea de que la igualdad en (1.1) es válida y que se propone, por ejemplo, el siguiente ejercicio combinado:

$$
\sqrt{4}-2 \cdot \sqrt{9} \cdot \sqrt{16}+3 \cdot \sqrt{25} \text {. }
$$

Por supuesto, nunca dudaríamos de lo que debemos hacer: separamos en términos y operamos. El resultado es -7 . Y acá aparece la primera inconsistencia ya que, si (1.1) es cierta y, en forma general, para cada $x>0$ se admiten dos resultados para $\sqrt{x}$, entonces debemos considerar los dos valores posibles en cada raíz cuadrada que aparece en el ejercicio dado. Así, si consideramos todas las posibles combinaciones de estos signos, el ejercicio no tiene un único resultado, ¡sino 16 posibles! Aunque resulta poco probable que un alumno de secundaria considere estas 16 opciones como resultado de un ejercicio combinado, lo mencionamos 
para enfatizar la inconsistencia que genera suponer dos valores posibles para el símbolo radical. Sin embargo, sí es más probable que un alumno se pregunte por qué la calculadora devuelve un solo resultado cuando se ingresa $\sqrt{4}$.

En algunas escuelas secundarias se aborda el concepto de función. Se habla de existencia y unicidad de imagen, se analizan gráficos de funciones y se puede presentar la función $f:[0, \infty) \rightarrow \mathbb{R}$ dada por $f(x)=\sqrt{x}$. Una forma usual de bosquejar la gráfica de dicha función es mediante una tabla de valores. ¿Qué valor le asignamos, por ejemplo, a $f(4)$ ? Si seguimos afirmando que la igualdad en (1.1) es cierta, entonces $f(4)= \pm 2$, es decir, no hay unicidad de imagen y por lo tanto no es función. Pero no, esto es incorrecto, ya que todos sabemos que $f$ es función y conocemos su gráfica (ver Figura 1). Este es otro ejemplo que pone en evidencia

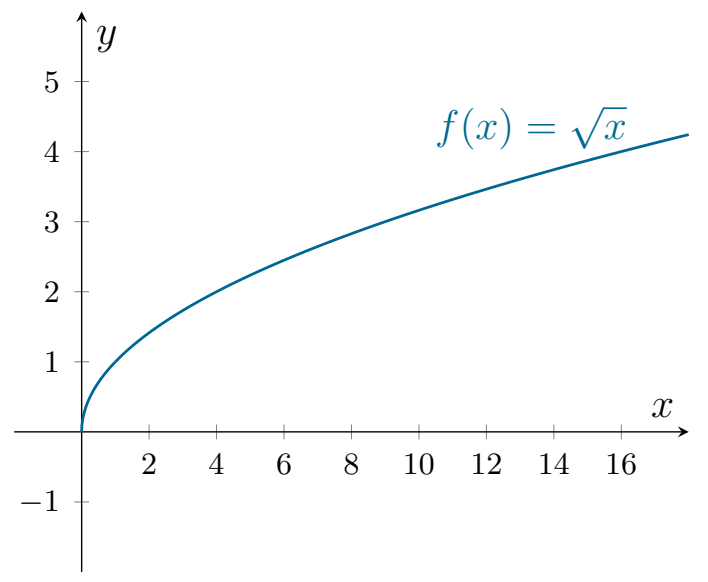

Figura 1. Gráfica de $f(x)=\sqrt{x}$.

las contradicciones que genera (1.1) alrededor de otros conceptos bien definidos.

Después de trabajar el concepto de función hay, al menos, dos tipos de funciones que merecen un trato especial: la afín y la cuadrática. Con respecto a esta última, es probable que se hable de su vértice, sus ramas, su eje de simetría, y sus raíces. Y es aquí donde la fórmula resolvente nos ayuda a calcular las raíces de la función $f(x)=a x^{2}+b x+c$, las cuales están dadas por

$$
x_{1,2}=\frac{-b \pm \sqrt{b^{2}-4 a c}}{2 a} .
$$

Y la pregunta natural es: si (1.1) fuera cierta, entonces la fórmula resolvente no necesitaría el doble signo antes de la raíz cuadrada, ya que ambos resultados estarían ya contemplados por la raíz. Si enseñamos que (1.1) vale, entonces debemos considerar correcto que un alumno afirme que la fórmula resolvente es

$$
x_{1,2}=\frac{-b+\sqrt{b^{2}-4 a c}}{2 a},
$$

ya que ambas opciones quedan implícitamente contenidas. 
Cabe señalar que el origen del doble signo antes de la raíz tiene su justificación en el uso de la propiedad $\sqrt{x^{2}}=|x|$, que trabajaremos con mayor detalle en la Sección 1.3, y en la definición del valor absoluto de un número, y no en pensar que $\sqrt{x}$ tiene dos valores posibles, cuando $x$ es un número real positivo.

Los ejemplos previos muestran algunas de las inconsistencias que puede generar en la construcción de otros conceptos el hecho de pensar que $\sqrt{4}= \pm 2 \mathrm{o}$, en forma general, que $\sqrt{x}$, con $x>0$, tiene dos resultados posibles. Así, independientemente del campo numérico en el cual se esté presentando la radicación (naturales, enteros o reales), el siguiente mensaje debe ser claro en la escuela secundaria:

Mensaje correcto 1. El símbolo $\sqrt{x}$, siendo $x$ un número real no negativo, tiene como resultado un único número real no negativo. Luego, $\sqrt{4}=2$.

Una pregunta natural en este punto es: ¿de dónde proviene el error de considerar dos resultados posibles? Proviene del uso del símbolo radical en la definición de raíz cuadrada de un número positivo. La siguiente definición, que resulta inconveniente por lo que mencionamos arriba, puede encontrarse en varios libros de Matemática escritos para la educación secundaria:

$$
\sqrt{x}=r \quad \text { si } \quad r^{2}=x .
$$

Si fuera correcta, entonces implicaría que $\sqrt{4}=2$ y también $\sqrt{4}=-2$, ya que tanto 2 como -2 satisfacen que al ser elevados al cuadrado dan 4 como resultado. Esto es, por supuesto, incorrecto ya que el símbolo $\sqrt{x}$ debe arrojar un único resultado para cada $x \geq 0$. Para evitarlo, se puede:

- Abordar, inicialmente, la enseñanza de las raíces cuadradas de un número $x$, sin usar el símbolo radical. Esto es: $r$ es una raíz cuadrada de $x$ si $r^{2}=x$.

- Luego, si $x \geq 0$, reservar el símbolo $\sqrt{x}$ solo para el resultado no negativo, es decir, para la llamada raíz cuadrada principal.

- Agregar el requisito mencionado en el inciso anterior dentro de la definición cuando se utilice el símbolo radical (Sobel y Lerner, 1996, p.38): si $x \geq 0$ entonces

$$
\sqrt{x}=r \quad \text { si } \quad r \geq 0 \quad \text { y } \quad r^{2}=x .
$$

Para el caso general, uno de los textos en donde la definición se encuentra sencilla y correcta es (Larson y Falvo, 2012). Allí, en su apéndice se define, para $x$ y $r$ números reales y $n \geq 2$ un entero positivo, los siguientes conceptos:

Raíz $n$-ésima de un número: $\operatorname{Si} r^{n}=x$ decimos que $r$ es una raíz $n$-ésima de $x$. 
Raíz $n$-ésima principal de un número: $\operatorname{Si} x$ tiene al menos una raíz $n$-ésima, se denomina principal a la que tiene el mismo signo que $x$. En tal caso, la misma se denota con el símbolo radical:

\section{$\sqrt[n]{x}$ denota la $n$-ésima raíz principal de $x$.}

Algo similar puede encontrarse en (Sobel y Lerner, 1996, p.38). Con esta definición queda claro que $\sqrt{4}= \pm 2$ es incorrecto, y que lo correcto es $\sqrt{4}=2$.

Si bien la demostración de la existencia de raíces cuadradas de números reales no negativos escapa a los contenidos de la escuela secundaria, la unicidad de $\sqrt{x}$ puede probarse fácilmente. En efecto, sea $x \geq 0$ y supongamos que existen dos números, $r$ y $a$, tales que $r=\sqrt{x}$ y $a=\sqrt{x}$. Entonces $r$ y $a$ son dos números reales no negativos tales que $r^{2}=x$ y $a^{2}=x$. Luego $r^{2}=a^{2}$, o bien

$$
r^{2}-a^{2}=0 .
$$

Factorizando, la ecuación anterior equivale a $(r-a)(r+a)=0$. Por la propiedad del producto cero* se tiene que

$$
r=a, \text { o bien } r=-a .
$$

Puesto que tanto $r$ como $a$ son números reales no negativos, lo anterior implica que $r=a$, lo que prueba la unicidad.

La existencia y unicidad mencionadas hacen que el símbolo radical pueda entenderse como una función definida en el dominio correspondiente, en el sentido que $\sqrt{x}$ asigna un único valor a cada $x \geq 0$.

\subsection{Radicación vs. ecuaciones.}

El mismo razonamiento aplicado para demostrar la unicidad de la radicación permite probar que las únicas soluciones de la ecuación

$$
x^{2}=4
$$

son $x=2$ y $x=-2$. En efecto, la ecuación equivale a resolver

$$
x^{2}-4=0,
$$

lo que a su vez es equivalente a $(x-2)(x+2)=0$. Nuevamente, por la propiedad del producto cero, se tiene que $x=2$ o $x=-2$. Así, el conjunto de soluciones de $x^{2}=4$ es $S=\{-2,2\}$.

Observar que el número 4 puede reemplazarse por cualquier otro número real positivo, es decir, de la misma forma se prueba que las únicas soluciones para la ecuación

$$
x^{2}=a,
$$

* Recordemos que esta propiedad establece que un producto de factores es igual a cero si y solo si uno o más de los factores es igual a cero. 
siendo $a$ un número real positivo, son $x=\sqrt{a}$ y $x=-\sqrt{a}$. Esto se resume escribiendo $x= \pm \sqrt{a}$ Ahora sí, el doble signo colocado delante del símbolo radical indica explícitamente que se deben considerar ambos valores. Así, el conjunto de soluciones de la ecuación $x^{2}=a$ es $S=\{-\sqrt{a}, \sqrt{a}\}$.

En relación a esto, haremos mención a un error de notación que puede generar confusión entre la operación radicación y el conjunto de soluciones de una ecuación. Precisamente, en (Covelo y Covelo, 2019, p.32) se indica que

$$
\sqrt{-4}=\emptyset \text {. }
$$

Lo anterior pretende transmitir que la raíz cuadrada de un número negativo no está definida en los números reales, asignando como resultado de la operación un conjunto: el vacío. De ninguna manera podemos asignar un conjunto como resultado a esta operación, en ningún campo numérico en el cual se esté trabajando la radicación. Así como no decimos que $\sqrt{4}=\{2\}$, no podemos decir que la raíz cuadrada de un número negativo es el conjunto vacío. Lo correcto es decir que no está definida en los números reales.

Mensaje falso 1. El símbolo $\sqrt{x}$ es un número en ciertos casos, pero es un conjunto en otros.

Aquí queda expuesta una confusión entre la operación radicación, cuyo resultado es un número o no está definida, y las soluciones de una ecuación, las cuales pueden expresarse como un conjunto. En particular, para el ejemplo mencionado se tiene que $\sqrt{-4}$ no está definida, mientras que el conjunto de soluciones de la ecuación

$$
x^{2}=-4
$$

es el vacío (es decir, no tiene solución).

Mensaje correcto 2. El símbolo $\sqrt{x}$ denota un único número real cuando $x \geq 0, y$ no está definido para $x<0$. Por otra parte, el conjunto $S$ de soluciones de la ecuación

$$
x^{2}=a
$$

es $S=\{-\sqrt{a}, \sqrt{a}\}$ cuando $a \geq 0$, y $S=\emptyset$ si $a<0$.

Queremos solamente observar, para concluir esta parte, que la forma de resolución de $x^{2}=a$ presentada previamente permite conocer, además, la multiplicidad de las raíces. Por ejemplo, consideremos la ecuación

$$
x^{4}=16 \text {. }
$$

Notar que la misma equivale a $x^{4}-16=0$. Aplicando diferencia de cuadrados se transforma en

$$
\left(x^{2}-4\right)\left(x^{2}+4\right)=0 .
$$


Por la propiedad del producto cero y lo trabajado antes, se concluye que el conjunto solución es $\{-2,2\} \cup \emptyset=\{-2,2\}$. La forma más frecuente de resolución de esta ecuación es la siguiente:

$$
\begin{aligned}
x^{4} & =16 \\
|x| & =\sqrt[4]{16}=2 \\
x & = \pm 2 .
\end{aligned}
$$

Más adelante volveremos sobre el valor absoluto involucrado en la resolución anterior. Si bien la misma es correcta, no da información sobre la multiplicidad de las raíces. Identificar de antemano la diferencia de cuadrados puede ayudar a no cometer errores al factorizar, en un futuro, expresiones como $x^{4}-16$ resolviendo la ecuación anterior, ya que el exponente de la variable suele hacer pensar al alumno que posee dos raíces dobles. Lo que sí resulta importante es no caer en el error de escribir una resolución como la siguiente:

$$
\begin{aligned}
x^{2} & =25 \\
x & =\sqrt{25},
\end{aligned}
$$

para luego deducir que $x=-5$ o $x=5$. En este proceso de resolución, que aparece en (Covelo y Covelo, 2020, p.76), se están cometiendo dos errores juntos: simplificar la raíz cuadrada con el exponente sin colocar el valor absoluto, y afirmar que $\sqrt{25}= \pm 5$.

\subsection{Algunas propiedades.}

Abordaremos ahora algunas propiedades muy conocidas de la radicación, de las cuales haremos uso a lo largo del texto. Previamente hicimos mención a la forma correcta de simplificar índice con exponente. Más precisamente, es sabido que para todo número real $x$ se tiene que

$$
\sqrt{x^{2}}=|x| \text {. }
$$

La prueba es sencilla y, por completitud, la incluimos a continuación.

Demostración. Sabemos que $\sqrt{x^{2}}$ denota al único número real $t \geq 0$ que verifica que $t^{2}=x^{2}$. Puesto que $|x|$ satisface ambas condiciones, esto es, es no negativo $\mathrm{y}$ $|x|^{2}=x^{2}$, entonces $t=|x|$, es decir, $\sqrt{x^{2}}=|x|$.

De la misma forma se prueba que $\sqrt[n]{x^{n}}=|x|$ para todo $n$ par y todo número real $x$, puesto que también ocurre que $|x|^{n}=x^{n}$ en dicho caso.

A pesar de que la prueba de la validez de (1.2) utiliza la definición correcta del símbolo radical, la misma no suele ser presentada para los alumnos de escuela secundaria. En general, es ilustrada mediante ejemplos de cómo debe aplicarse. Así, 
contamos con alumnos que, aún aplicando de manera acertada (1.2), la concepción incorrecta de los dos resultados posibles para el símbolo radical los conduce a cometer un error en la resolución de desigualdades simples como

$$
x^{2} \leq 4 \text {. }
$$

Concretamente, un grupo de alumnos de tercer año acudió a su profesor, autor de este trabajo, con el siguiente planteo: para resolver la desigualdad anterior aplicaron raíces cuadradas a ambos miembros y la monotonía de la raíz (es monótona creciente, por lo que conserva el sentido de la desigualdad) para obtener:

$$
\sqrt{x^{2}} \leq \sqrt{4}
$$

Luego, al emplear el concepto erróneo que $\sqrt{4}= \pm 2$, concluyeron que:

$$
|x| \leq \pm 2 \text {. }
$$

Así, se llega a una desigualdad que no tiene sentido y que no refleja la solución buscada. Esto no habría ocurrido si siempre se hubiera tenido en claro que el símbolo denota la raíz principal, obteniendo así:

$$
|x| \leq 2
$$

es decir, $-2 \leq x \leq 2$.

\section{$\S 2$. Los exponentes racionales y las bases negativas}

En esta sección abordaremos el problema de trabajar con potencias con bases negativas y exponente racional. Precisamente, veremos cómo los obstáculos se generan desde la definición y el uso de las propiedades.

\subsection{La definición}

La radicación es la operación que permite definir la potencia con exponente racional. El camino para ello es el siguiente:

(d.1) Se define la potencia con base $x \in \mathbb{R}$ y exponente natural $n$ como el producto de $x \cdot x \cdot \ldots \cdot x$, donde $x$ aparece como factor $n$ veces.

(d.2) Se define $x^{0}=1$, siempre que $x \neq 0$.

(d.3) Para $p, q \in \mathbb{N}$ y $x \in \mathbb{R}$, se define

$$
x^{\frac{p}{q}}=\sqrt[n]{x^{m}}
$$

en caso que $\sqrt[n]{x^{m}}$ esté definida, siendo $m / n$ la forma irreducible de $p / q$.

(d.4) Si $b$ es un número racional positivo, se define $x^{-b}$ como $\frac{1}{x^{b}}$, siempre que $x^{b}$ sea un número real distinto de cero. En particular, se tiene que $x^{-n}=\frac{1}{x^{n}}$ para todo $n \in \mathbb{N}$ y $x \neq 0$. 
Así, tenemos por ejemplo que

$$
(-8)^{\frac{2}{6}}=(-8)^{\frac{1}{3}}=\sqrt[3]{-8}=-2 .
$$

Sucede que, en general, el requisito de utilizar la forma irreducible para el exponente no está contemplado en los libros de texto utilizados en la enseñanza secundaria. En ellos, lo que suele encontrarse como definición en lugar de (d.3) es lo siguiente:

(d.5) Si $m \in \mathbb{Z}$ y $n \in \mathbb{N}$, se define

$$
x^{\frac{m}{n}}=\sqrt[n]{x^{m}}
$$

en caso de que sea posible.

Veamos qué ocurre si aplicamos esta definición para calcular lo siguiente:

$$
(-8)^{\frac{1}{3}}=\sqrt[3]{-8}=-2
$$

pero si escribimos $\frac{1}{3}$ de manera equivalente como $\frac{2}{6}$, entonces:

$$
(-8)^{\frac{1}{3}}=(-8)^{\frac{2}{6}}=\sqrt[6]{(-8)^{2}}=2 .
$$

Así, vemos que se obtienen diferentes resultados al utilizar distintas representaciones del mismo exponente racional.

En matemática, que un concepto esté bien definido significa que se determina sin ambigüedad alguna, es decir, que no depende de la representación o forma de llegar a los objetos involucrados. Si se enuncia una definición por una elección arbitraria, entonces se debe comprobar que la definición es independiente de dicha elección. El ejemplo anterior muestra que la definición (d.5) no es buena si permitimos que la base sea negativa y, sin embargo, es la más frecuente en los libros de Matemática de nivel secundario. Aunque en los ejercicios no aparecen, en general, exponentes racionales no irreducibles, los mismos suelen generarse cuando se aplican propiedades de la potencia (como productos de potencias de igual base o potencia de otra potencia). Analizaremos con mayor profundidad lo referido a la validez de las propiedades en la subsección siguiente.

Mensaje contradictorio 1. La definición (d.5) de potencia con exponente racional no puede contemplar bases negativas, ipero éstas siempre aparecen en los ejercicios!

Por supuesto que la condición de ser el exponente irreducible no es necesaria si solamente trabajamos con bases positivas. En dicho caso, es fácil ver que la definición no depende de la representación elegida para el racional (ver, por ejemplo, (Noriega, 1979)). Sin embargo, si se quiere abarcar tanto bases positivas como negativas, esta condición garantiza la buena definición de potencias con exponente racional. 
Es importante también remarcar que, aún considerando solo bases positivas, los requisitos $m \in \mathbb{Z}$ y $n \in \mathbb{N}$ son importantes cuando se define $x^{\frac{m}{n}}=\sqrt[n]{x^{m}}, \mathrm{y}$ esto no siempre es mencionado en los textos escolares. En efecto, si se enuncia solamente $\frac{m}{n} \in \mathbb{Q}$, se permite que el denominador sea un entero negativo, y la definición no tiene sentido pues la radicación no se define para índices negativos. Así, si el exponente es un racional negativo, el signo menos lo debe llevar el numerador, para poder aplicar la definición para exponentes enteros negativos y luego la radicación. Este requisito sobre el numerador y el denominador de un exponente racional no suele ser explicitado en la mayoría de los libros de Matemática utilizados en la escuela secundaria.

Mensaje correcto 3. Las condiciones sobre el exponente impuestas en (d.3) garantizan la correcta definición de potencias con exponente racional, incluyendo a las bases negativas.

Pese a que queda demostrada la necesidad de requerir el exponente en su forma irreducible, esta condición está ausente en gran parte de los libros de texto. Sin embargo, aunque no está escrito, cuando en (De Simone y Turner, 2016, p.19) se afirma que "si $a$ es un número real negativo, la definición $a^{\frac{m}{n}}=\sqrt[n]{a^{m}}$ tiene sentido sólo si $n$ es impar", pareciera estar considerando la condición de irreducibilidad de $m / n$. De lo contrario, dicha afirmación es falsa, pues basta con observar que

$$
(-1)^{\frac{4}{6}}=\sqrt[3]{(-1)^{2}}=1
$$

y también

$$
\sqrt[6]{(-1)^{4}}=\sqrt[6]{1}=1
$$

por lo que es cierta la igualdad $(-1)^{4 / 6}=\sqrt[6]{(-1)^{4}}$, a pesar de que $a=-1$ es negativo y $n=6$ es par. Esto muestra, una vez más, que dicha condición debe explicitarse si se quiere trabajar con bases negativas.

Es importante también mencionar que la potencia con exponente racional se define a través de la radicación. Este procedimiento no puede quedar plasmado como un simple intercambio de números para pasar de una potencia a una radicación, y viceversa. No son, a priori, dos escrituras equivalentes, sino la definición de una operación a partir de otra ya existente. No se debe confundir al alumno tratando de demostrar, como en (Kaczor, Schaposchnik, Franco, Cicala, y Díaz, 1999, p.37), que $x^{\frac{m}{n}}=\sqrt[n]{x^{m}}$, ya que es exactamente su definición. Tampoco se debe expresar, como en (Covelo y Covelo, 2020, p.48), que "las raíces también se pueden expresar como potencias: $\sqrt[a]{b^{c}}=b^{\frac{c}{a}}$ ". Por supuesto que esta igualdad es verdadera pero, justamente, porque el miembro derecho se define como el izquierdo, y no al revés.

Para finalizar esta parte, cabe señalar que en (Sobel y Lerner, 1996, p.41) se da una definición alternativa de potencias con exponente racional, donde cambia la condición de irreducible en el exponente por otra: 
(d.6) Sean $m, n \in \mathbb{N}$ y sea $x \in \mathbb{R}$. Se define

$$
x^{\frac{m}{n}}=\sqrt[n]{x^{m}}=(\sqrt[n]{x})^{m}
$$

siempre que $\sqrt[n]{x}$ esté definida.

Esta última condición, que es distinta a pedir que $\sqrt[n]{x^{m}}$ esté definida como en (d.3), hace que no se presenten ambigüedades en la definición ya que no podemos aplicarla para calcular $(-8)^{\frac{2}{6}}$, debido a que $\sqrt[6]{-8}$ no está definida. Entonces no queda otra opción que aplicar la definición con su exponente reducido a $\frac{1}{3}$, o con cualquier otra fracción equivalente cuyo denominador no sea par. Así, con esta definición, tenemos:

$$
(-8)^{\frac{1}{3}}=\sqrt[3]{-8}=-2, \quad(-8)^{\frac{2}{6}}=(-8)^{\frac{1}{3}} .
$$

Además, otra observación en cuanto a la no ambigüedad de la definición (d.6) es que si $m / n=p / q$ y además están definidas tanto $\sqrt[n]{x^{m}}$ como $\sqrt[q]{x^{p}}$, entonces obtendremos el mismo valor en el lado derecho de (d.6) usando cualquiera de las representaciones. Por ejemplo:

$$
(-8)^{\frac{1}{3}}=\sqrt[3]{-8}=-2, \quad(-8)^{\frac{3}{9}}=\sqrt[9]{-512}=-2 .
$$

Puesto que todo el análisis previo presenta demasiada complejidad para ser abordado en la escuela secundaria, restringirse a bases positivas parece una buena opción para abordar este tema. El modo en que se trabaja actualmente, que incluye a las bases negativas pero no se aclara nada, conduce a las ambigüedades enunciadas y a los errores y contradicciones producidos por la aplicación de propiedades que expondremos a continuación.

\subsection{Las propiedades}

Una vez definida la radicación, analicemos las consecuencias de permitir el uso de las propiedades de la potencia cuando la base es negativa. Es común encontrar en la bibliografía ejercicios del tipo: Resolver aplicando propiedades de la potenciación:

$$
\begin{aligned}
& \text { i. }(-27)^{\frac{1}{3}}:(-27)^{-1} \cdot(-27)^{\frac{2}{3}}:(-27)^{-2}= \\
& \text { ii. }\left(a^{m}\right)^{\frac{1}{n}}=
\end{aligned}
$$

Así, el mensaje es claro: las propiedades de la potencia pueden usarse también con bases negativas. Es cierto que en el primer inciso se tuvo el cuidado de que cada vez que aparece un exponente racional, su denominador sea impar. Pero, en general, no se hace referencia a ello. En el segundo, al no decir nada sobre $a$, se entiende que puede ser cualquier número real. ¿Qué respuesta se espera en este último caso? Si se espera que la respuesta sea:

$$
\left(a^{m}\right)^{\frac{1}{n}}=a^{\frac{m}{n}},
$$


entonces se debe considerar correcto si un alumno realiza el siguiente procedimiento:

$$
\sqrt{(-3)^{2}}=\left[(-3)^{2}\right]^{\frac{1}{2}}=(-3)^{\frac{2}{2}}=(-3)^{1}=-3,
$$

aunque contradiga (1.2), ya que es la misma resolución que la efectuada para ii), con $a=-3$ y $m=n=2$.

Esto conduce a que el alumno obtenga un resultado si aplica las propiedades, diferente al obtenido al utilizar la fórmula (1.2) previamente trabajada:

Mensaje contradictorio 2. $\left(a^{m}\right)^{\frac{1}{n}}=a^{\frac{m}{n}}$ pero $\left(a^{2}\right)^{\frac{1}{2}}=|a|$. Entonces, ¿corresponde o no el valor absoluto?

El origen de esta ambigüedad es, sin dudas, el mensaje que se deja en el alumno al presentarle ejercicios donde deben aplicar las propiedades de la potencia con exponentes racionales y bases negativas.

Mensaje falso 2. Las propiedades de la potencia estudiadas para exponentes enteros pueden extenderse a exponentes racionales, sin aclaración adicional.

A diferencia del caso de exponentes enteros, donde las propiedades valen independientemente del signo de la base, estas no valen, en general, cuando la base es negativa y el exponente es racional. Esto se prueba observando los siguientes ejemplos:

$$
\begin{aligned}
& \left((-4)^{2}\right)^{\frac{1}{4}} \neq(-4)^{\frac{1}{2}}, \\
& -1 \neq(-1)^{\frac{1}{2}} \cdot(-1)^{\frac{1}{2}} .
\end{aligned}
$$

Lo primero ilustra que no vale la propiedad llamada "potencia de otra potencia" que enuncia que $\left(x^{a}\right)^{b}=x^{a b}$, mientras que la segunda invalida la conocida como "producto de potencias de igual base", que asegura que $x^{a} \cdot x^{b}=x^{a+b}$. Recordemos que estamos trabajando en los números reales (las operaciones en los números complejos merecen un análisis aparte). De hecho, aún cuando no aparezcan raíces con índice par y radicando negativo, las propiedades no valen: por ejemplo, por un lado tenemos que

$$
\left((-8)^{\frac{2}{3}}\right)^{\frac{1}{2}}=4^{\frac{1}{2}}=2
$$

mientras que, por el otro,

$$
(-8)^{\frac{2}{3} \cdot \frac{1}{2}}=(-8)^{\frac{1}{3}}=-2 .
$$

Nuevamente, vemos que $\left(x^{a}\right)^{b}$ es distinto de $x^{a b}$.

Observar que el inconveniente aparece cuando se involucra un exponente racional con denominador par. Sin embargo, en la mayoría de la bibliografía no se dice nada sobre esto, y las propiedades se aplican sin pedir que la base sea positiva. Restringirse solamente a bases positivas vuelve a aparecer como opción para trabajar correctamente las propiedades de la potencia con exponente racional. Frente 
a esta elección, es importante enfatizar y explicitar que las propiedades de la potenciación de exponentes racionales sí son válidas cuando la base es positiva, pues se deducen de su definición y de las propiedades de la radicación.

Lo que sí podemos decir es que las propiedades valen siempre y cuando cada una de las cantidades que intervienen estén definidas. Por ejemplo, si $a$ y $b$ son racionales, entonces:

$$
x^{a} \cdot x^{b}=x^{a+b}
$$

siempre que $x^{a}, x^{b}$ y $x^{a+b}$ sean número reales, lo que ocurre, por ejemplo, cada vez que el exponente es un racional con denominador impar, independientemente del signo de $x$. Lo mismo para la resta. Para el caso de potencia de otra potencia, debido a la propiedad conmutativa del producto, se debe requerir que las cantidades involucradas existan en ambos órdenes: $\left(x^{a}\right)^{b}=x^{a b}=\left(x^{b}\right)^{a}$, siempre que cada una de las cantidades $x^{a}, x^{b}$ y $x^{a b}$ sea un número real. La validez de todo lo anterior puede demostrarse, por ejemplo, utilizando la fórmula (2.1) dada posteriormente en este trabajo, pero escapa a los contenidos de la escuela secundaria.

El hecho de transmitir que las propiedades de las potencias pueden aplicarse para exponentes racionales sin decir nada sobre la base, además de producir errores como todos los ya enunciados, contradice observaciones en las cuales se pone mucho énfasis al enseñar radicación. Más precisamente, se aclara insistentemente que la propiedad distributiva de la radicación con respecto a la multiplicación y a la división es válida siempre que existan cada una de las raíces por separado, es decir, siempre que cada una sea un número real:

$$
\sqrt{(-25) \cdot(-4)}=10 \neq \sqrt{-25} \cdot \sqrt{-4},
$$

lo cual es correcto, pero no siempre se aplica la misma rigurosidad cuando se piensa el mismo ejercicio como potencias con exponentes racionales.

Mensaje contradictorio 3. Si bien $x^{\frac{1}{2}} y \sqrt{x}$ se presentan como equivalentes, las restricciones en el uso de las propiedades no siempre se plantean para la potencia pero sí para la radicación.

Para lograr una enseñanza consistente resulta necesario, aunque no suficiente, transmitir que las propiedades de la potencia valen "siempre y cuando cada una de las cantidades que intervienen estén definidas", como ya mencionamos previamente. Notar que esta frase tiene la simpleza suficiente como para ser presentada en el nivel secundario, en caso de que se desee (o se necesite) trabajar con bases negativas y exponentes racionales.

Mensaje correcto 4. Las propiedades de la potencia no valen, en general, cuando la base es negativa y el exponente es racional. Sin embargo, pueden aplicarse en tal caso cuando cada una de las cantidades que intervienen estén definidas en los reales. 
Finalizamos esta parte exponiendo más obstáculos generados en el aprendizaje de contenidos básicos de la escuela secundaria, al permitir el uso de las propiedades de la potencia con exponente racional y base negativa. En particular, en la extracción de factores fuera del signo radical.

Este contenido está estrechamente ligado con el uso de las propiedades de la potenciación y la radicación. Más precisamente, las propuestas editoriales hacen su aporte en este estudio de los radicales ejemplificando cómo usar dichas propiedades para lograr ese objetivo.

El esfuerzo está puesto en mostrar la técnica para lograr extraer correctamente factores y se descuida el análisis sobre las variables involucradas en el radicando. Por ejemplo, en (De Simone y Turner, 2016, p.13), se ejemplifica cómo extraer factores en el siguiente caso:

$$
\sqrt{12 a^{3} b^{2}}=\sqrt{2^{2} 3 a^{2} a b^{2}}=2 a b \sqrt{3 a} .
$$

En lo anterior, no se especifica nada sobre la naturaleza de $a$ y $b$. Es claro que $a$ no puede ser negativo puesto que, en tal caso, el radicando inicial sería negativo. La pregunta natural es: ¿qué ocurre si $b$ es negativo? Por ejemplo, utilicemos la igualdad obtenida con $a=1$ y $b=-1$ :

$$
\sqrt{12}=-2 \sqrt{3}
$$

lo cual es, claramente, incorrecto. El error se podría haber evitado de dos maneras:

- la primera es restringir el ejercicio propuesto en el texto mencionado solamente a valores no negativos para $a$ y $b$;

- la segunda, que permite que $b$ sea cualquier número real, es trabajar de modo correcto la simplificación de índices con exponentes, utilizando que $\sqrt{b^{2}}=|b|$. Así, la fórmula correcta para $a \geq 0 \mathrm{y} b \in \mathbb{R}$, es

$$
\sqrt{12 a^{3} b^{2}}=2 a|b| \sqrt{3 a} \text {. }
$$

Sin embargo, nuevamente un alumno podría llegar a una fórmula incorrecta y diferente a la anterior si comete el error de aplicar las propiedades de la potencia para bases que pueden ser negativas:

$$
\sqrt{12 a^{3} b^{2}}=\sqrt{3 \cdot 2^{2} a^{3} b^{2}}=\left(3 \cdot 2^{2} a^{3} b^{2}\right)^{\frac{1}{2}}=3^{\frac{1}{2}} 2 a^{\frac{3}{2}} b=2 a b(3 a)^{\frac{1}{2}}=2 a b \sqrt{3 a} .
$$

Por tal motivo, hacemos énfasis nuevamente en el error grave que se comete al desarrollar el tema incluyendo bases negativas sin aclaración alguna. 


\subsection{Una alternativa}

Una forma para no cometer errores que conduzcan a resultados incorrectos al trabajar con bases negativas y exponentes racionales es aplicar la siguiente fórmula (ver, por ejemplo, Carena, 2019, p.29) que relaciona una potencia con base negativa con otra cuya base es positiva, siempre que el exponente sea irreducible y tenga denominador impar (el cual es el único caso que importa pues, de lo contrario, la potencia no existe). Más precisamente, sean $m$ y $n$ naturales, con $n$ impar y $\frac{m}{n}$ irreducible. Para $x>0$ se tiene que

$$
(-x)^{\frac{m}{n}}=(-1)^{m} x^{\frac{m}{n}}
$$

La demostración de la fórmula (2.1) es sencilla debido a las hipótesis: aplicando (d.3) tenemos que $(-x)^{\frac{m}{n}}=\sqrt[n]{(-x)^{m}}$. Además,

$$
(-1)^{m}=\left\{\begin{aligned}
1, & \text { si } m \text { es entero par; } \\
-1, & \text { si } m \text { es entero impar. }
\end{aligned}\right.
$$

Lo anterior, junto a la definición de raíz $n$-ésima, implican que $\sqrt[n]{(-1)^{m}}=(-1)^{m}$, para todo entero $m$ y todo $n$ natural impar. Luego, aplicando la propiedad distributiva de la raíz con respecto al producto (recordar que es posible porque el índice es impar), se tiene que

$$
(-x)^{\frac{m}{n}}=\sqrt[n]{(-x)^{m}}=\sqrt[n]{(-1)^{m} x^{m}}=\sqrt[n]{(-1)^{m}} \sqrt[n]{x^{m}}=(-1)^{m} x^{\frac{m}{n}}
$$

como queríamos ver.

Es justamente la fórmula (2.1) la que permite probar que las propiedades de la potencia valen para cualquier base siempre que cada una de las cantidades involucradas exista, pero también es una herramienta muy útil para "deshacernos" de las bases negativas. De esta forma cada potencia con exponente racional se transforma en una potencia con base positiva y exponente racional, multiplicada por una potencia de base -1 y exponente entero. En cada uno de estos dos casos, las propiedades de la potencia pueden ser aplicadas.

\section{Conclusiones y reflexiones finales}

Lo expuesto en este trabajo nos recuerda que todo material bibliográfico puede contener errores. Es importante desde nuestro rol docente estar atentos a ello ya que, como vimos, pequeños detalles de enunciado, definición o notación pueden crear pensamientos contradictorios y erróneos en los alumnos. Esto influye de forma negativa en el aprendizaje, desde lo conceptual hasta en el interés por la disciplina. 
En este punto, nos parece importante y pertinente recordar, nuevamente, palabras enunciadas en (Bidart Gauna y cols., 2017), trabajo en el que se aborda un conflicto similar al aquí planteado pero en relación a la racionalización:

La situación brevemente descrita a lo largo de este artículo nos lleva a la encrucijada de estar frente a un obstáculo tanto didáctico como epistemológico muy evidente. Siendo que estamos frente a él, ¿no vale la pena corregirlo? Sin dudas, nos arriesgamos a pensar que sí. [...] La idea de corregir este concepto, más allá de que debemos hacerlo, se basa en el hecho de que instalarlo de manera errónea nos provoca, no sólo a nosotros, los docentes, sino también a los alumnos, un doble trabajo, innecesario por cierto, en el proceso de enseñanza y aprendizaje, ya que los errores instalados son resistentes y difíciles de superar porque esto requiere una reorganización de los conocimientos que el alumno y los docentes poseemos. Debemos comenzar a discutir entonces qué acontece en los espacios de formación, qué tipo de trabajo se despliega en torno a lo matemático que lleva a que ciertas ideas erróneas perduren a lo largo de los años. (p.20)

La comunicación de conceptos de forma precisa y correcta es una condición necesaria, aunque no suficiente, para un aprendizaje sólido. En caso de observar entre los alumnos la presencia de conceptos erróneos, será precisó revisarlos a fin de aclararlos y corregirlos, para así lograr una construcción consistente. Como menciona la cita previa, esto provoca un doble trabajo, tanto para los docentes como para los alumnos, ya que ciertos errores instalados son resistentes y persistentes. En muchos casos, como en el de la radicación, este camino puede agilizarse al iniciarse con la concepción correcta del símbolo radical desde su aparición.

En el caso particular de la potencia con exponente racional, una opción posible es restringirse a las bases positivas, donde sí valen las propiedades de las potencias. Es preciso evaluar si es conveniente utilizar bases negativas al momento de afianzar el uso de dichas propiedades mediante ejercitación, teniendo en cuenta el gran riesgo de incurrir, por un lado, en errores matemáticos no menores y, por el otro, en la transmisión de contradicciones o inconsistencias. En caso de ser necesario trabajar con bases negativas y exponentes racionales se deberían, entonces, incorporar las condiciones y advertencias que garanticen una construcción matemática consistente. Este trabajo pretende, a su vez, reunir ejemplos que sirvan para exponer las contradicciones y resultados incorrectos que surgen al no imponer dichas condiciones, para que puedan ser utilizados como herramientas en el proceso educativo.

Agradecimientos. Los autores agradecen las observaciones y sugerencias realizadas tanto por el editor como por los/as evaluadores/as de este artículo, quienes reflejaron en sus reportes el tiempo y seriedad que dedicaron a la lectura y mejora 
del mismo. Además, se agradecen los comentarios de Estefanía Dalmasso, Ignacio Garcia y Victoria Gómez durante la elaboración de este trabajo.

\section{Bibliografía}

Bidart Gauna, G., Cabral, G., Cafure, A., Cambriglia, V., y Fuentes, C. (2017). Algunas reflexiones sobre la racionalización. Rev. Educ. Mat, 32(1), 9-21.

Carena, M. (2019). Manual de matemática preuniversitaria. Ediciones UNL, Santa Fe.

Covelo, L., y Covelo, M. E. (2019). Matemática 2. Maipue, Buenos Aires.

Covelo, L., y Covelo, M. E. (2020). Matemática 3. Maipue, Buenos Aires.

De Simone, I., y Turner, M. (2016). Matemática, funciones y estadísticas. A-Z editora, Buenos Aires.

Ferraris, L., y Tasso, M. (2008). Aprendamos matemática 8. ComunicArte, Córdoba.

Kaczor, P., Schaposchnik, R., Franco, E., Cicala, R., y Díaz, B. (1999). Matemática 1. Ed. Santillana, Buenos Aires.

Larson, R., y Falvo, D. (2012). Precálculo, octava edición. CENGAGE Learning, México.

Noriega, R. (1979). El número real. Ed. Docencia, Buenos Aires.

Pacetti, A., y Bonardi, C. (2016). Matemática 3. El semáforo, Córdoba.

Sobel, M., y Lerner, N. (1996). Álgebra. Pearson - Prentice Hall.

FERNANDo BeniteZ

Escuela de Educación Secundaria Orientada Particular Incorporada Nro. 8132 "Belén"

(凶) benitezfer10@gmail.com

Marilina Carena

CONICET - Facultad de Ingeniería Química (UNL)

(৫) marilcarena@gmail.com

Recibido: 18 de abril de 2020.

Aceptado: 9 de enero de 2021.

Publicado en línea: 5 de abril de 2021. 